\title{
AN INTRODUCTION TO QUANTUM FIELD THEORY
}

\author{
by Michael E. Pestin and Daniel V. Schroeder
}

A BOOK REVIEW

\author{
CHRISTIAN GÜNTHER \\ P.O. Box 1465 \\ Libby, MT 59923 USA
}

(Received September 1996; Revised January 1997)

Quantum Field Theory arose as an attempt to unify quantum mechanics, classical fields theory, and relativity. These goals have been reached only partially and a rigorous foundation of the theory is still missing. However, Quantum Field Theory turned out to be extremely successful in all physical applications: nuclear physics, the theory of matter, elementary particles, high energy physics, and astrophysics. The success of the theory is so overwhelming that - even with shaky foundations and many areas still mathematically and conceptionally unresolved - there is no doubt that Quantum Field Theory has evolved into a mature subject with a core of established permanent results.

Textbooks are good indicators for the state of a theory. Of the many good textbooks on Quantum Field Theory, the book under review reflects the maturity of the field: this book manages to represent the core of Quantum Field Theory as a logical and transparent subject.

The book is organized in three parts, which cover much of the established material of Quantum Field Theory and standard particle theories.

The first part contains the general motivation, fundamental materials, and free quantum fields. A very short section on classical field theory is followed by the quantization of the Klein-Gordon field. The authors adopt a purely field-theoretic point of view avoiding the discussion about second quantization. Field quantization is introduced in the usual ad hoc method by writing down the operator valued "equal time" commutation relations. However the concepts of creation/annihilation operators, particles as field excitations with interpretation of position and momentum operators are carefully spelled out and the Lorentz invariance of these concepts is shown. Causality, Propagators and particle creation finish this the treatment of the Klein Gordon field. The Dirac field is discussed with similar diligence paying particular attention to internal symmetries.

After introducing free fields, the book turns to the study of renormalizable interacting fields: The authors start this study with a short discussion on renormalizability and then discuss Perturbation, Wicks theorem, S-Matrix, and Feynman rules. The following chapters treat quantum electrodynamics and radiation in detail. This in- 
cludes discussions of Compton scattering, infrared divergences, LSZ theory, etc.

The middle part of the book is devoted to renormalization. This part begins with an introduction to functional integration and Maxwell and spinor field quantization. Chapters on Classification of renormalization and application in $\phi^{4}$ and QED, on spontaneous symmetry braking, the renormalization group and Wilson's approach to renormalization (relationship with statistical mechanics) with applications to phase transitions and condensed matter systems follow.

The final part of the book covers Non Abelian Gauge Theories and provides the materials for the standard particle models. A short introductory chapter on gauge invariance and Yang-Mills fields is followed by the chapters on Quantization of NonAbelian Gauge Fields, Quantum Chromodynamics, and applications. Gauge fields with Spontaneous symmetry braking and weak interactions and, finally, quantization of spontaneously broken gauge fields follow.

The text is well organized and clearly written. Clarity in (physical) concept and style gives the reader the impression to be on firm ground. The book is rich in applications and practical calculations that are explicitly worked out throughout the text. Readers should have background in theoretical mechanics, electrodynamics, and Quantum mechanics, as well as in some elementary tensor analysis and transformation groups.

Problem sections at the end of each chapter provide more insights into the concepts and applications. A major "project" is found after each of the three main parts.

The covered material is mostly standard and the text uses classical mathematical language. This "conservative" approach could let the subject sometimes appear more settled than it is. Elementary calculations at times tend to hide the underlying mathematical structures and here and there intuitive arguments replace rigid proofs. Global topological aspects of gauge theory are not mentioned, and there is no hint to the problems of the foundations of the theory or to any alternative approaches. A short bibliography with comments give hints for more critical studies. There is no detailed reference section.

With this book, the authors have mostly succeeded in their goal to make a difficult and still unsettled subject accessible to students and non-specialists. It is a valuable addition to the current literature on this field and it can be warmly recommended as an (even affordable) textbook for a course or for self study.

\author{
AN INTRODUCTION TO QUANTUM FIELD THEORY \\ by Michael E. Peskin and Daniel V. Schroeder \\ Publisher Addison-Wesley \\ USA: One Jacob Way, Reading, MA 01867 \\ Publication Year 1995 \\ ISBN 0-201-50397-2 \\ Price: $\$ 54.82$
}




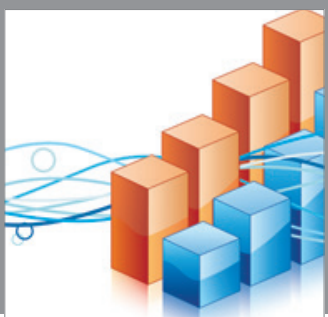

Advances in

Operations Research

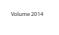

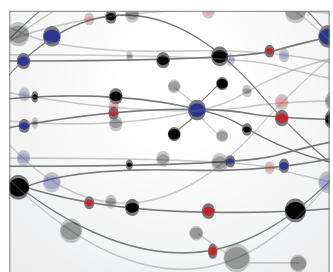

\section{The Scientific} World Journal
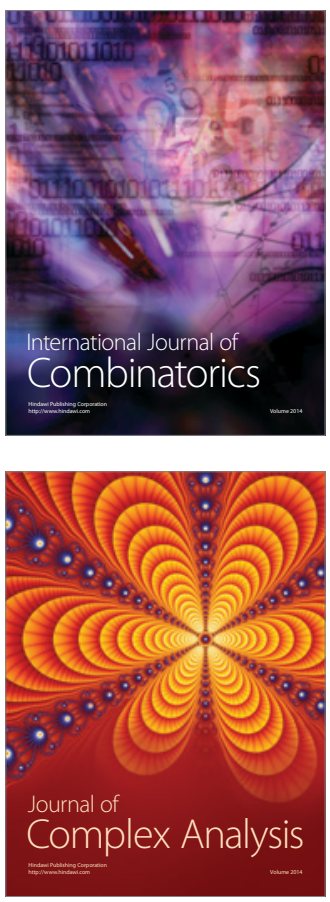

International Journal of

Mathematics and

Mathematical

Sciences
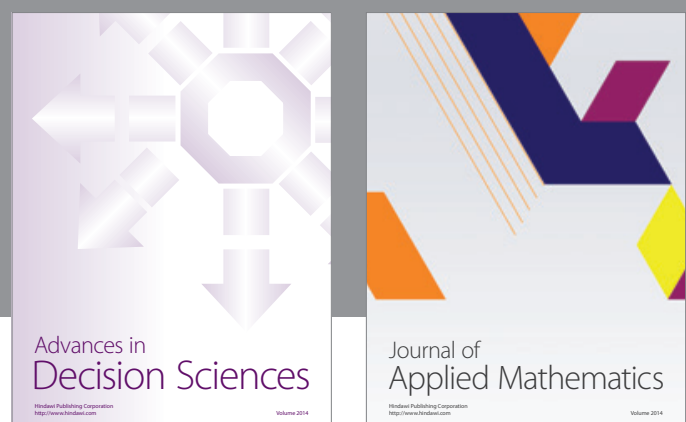

Journal of

Applied Mathematics
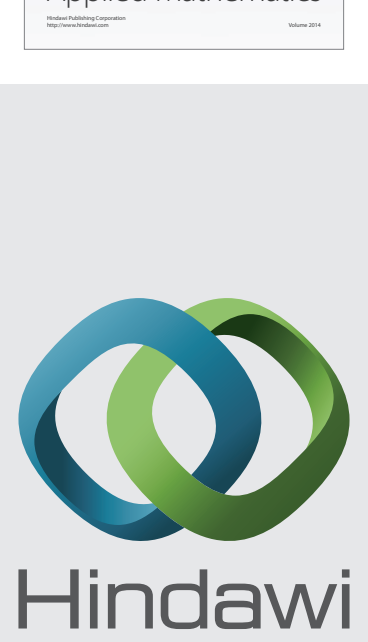

Submit your manuscripts at http://www.hindawi.com
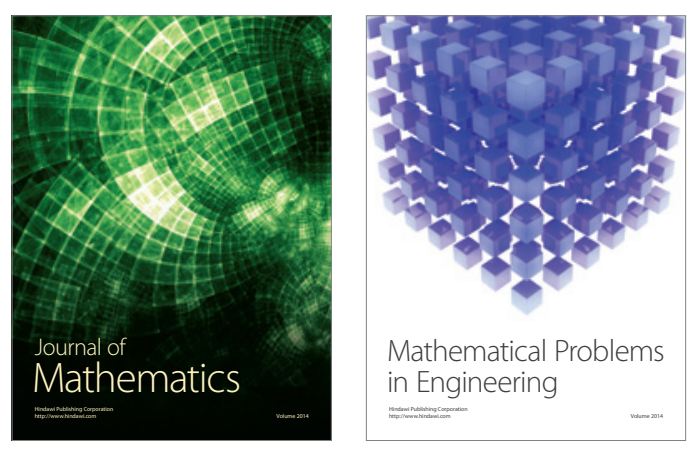

Mathematical Problems in Engineering
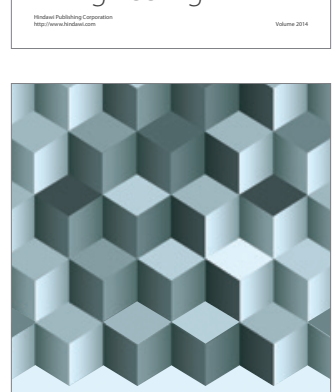

Journal of

Function Spaces
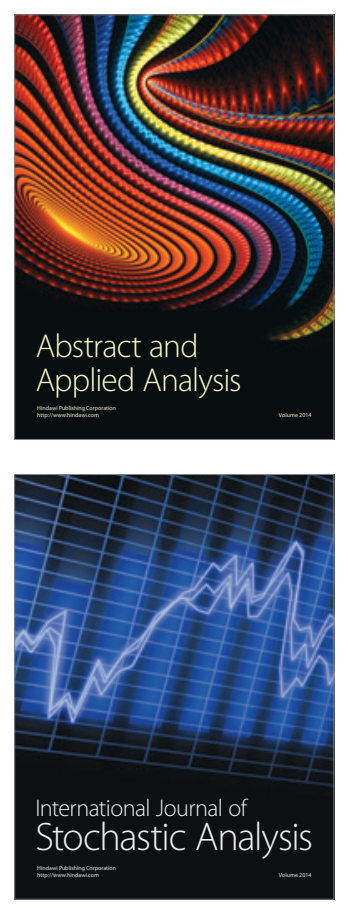

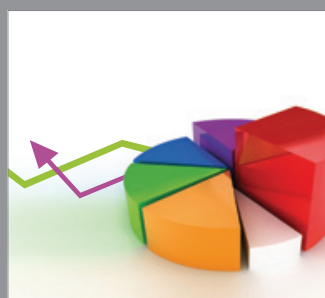

ournal of

Probability and Statistics

Promensencen
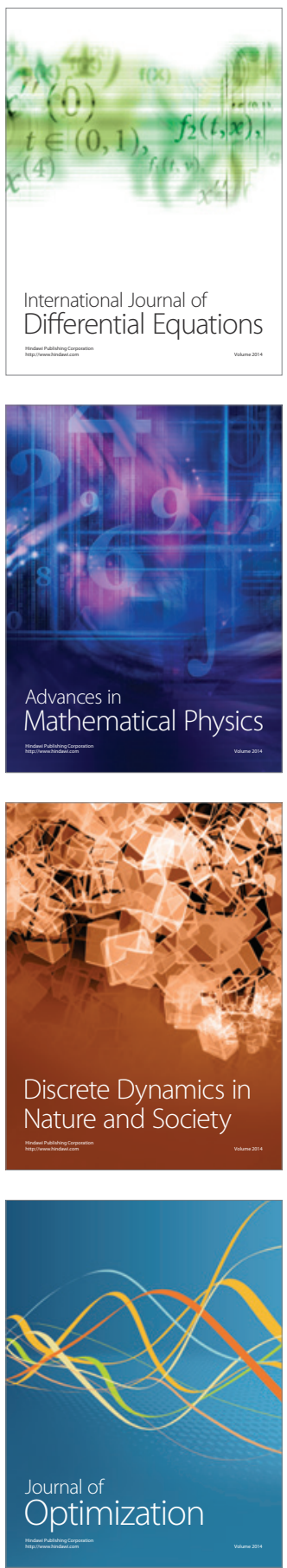